\title{
Lead fracture of peripheral nerve stimulator for brachial plexopathy -a case report-
}

\author{
Shu Chung Choi ${ }^{1}$, Ji Seon Chae ${ }^{2}$, Youn Jin $\mathrm{Kim}^{2}$, Jin Young Chon ${ }^{1}$, \\ and Ho Sik Moon ${ }^{1}$ \\ Department of Anesthesiology and Pain Medicine, ${ }^{1}$ College of Medicine, The Catholic University of Korea, ${ }^{2}$ College \\ of Medicine, Ewha Womans University, Seoul, Korea
}

Peripheral nerve stimulation (PNS) is a useful treatment for chronic pain, but it can cause damage depending on its application site. Here, we describe the case of a 54-year-old man who underwent PNS for brachial plexopathy in 2015. One lead was implanted on the left medial cord to stimulate the medial antebrachial cutaneous nerve, and the other was implanted on the radial nerve to stimulate the posterior antebrachial cutaneous nerve. Both leads were inserted near the shoulder joint but did not cross it. Before PNS, the patient did not move his shoulder and elbow because of severe pain, but the treatment greatly alleviated this pain. Twenty months after the operation, both leads were fractured, and the severe pain returned. Repetitive motion near the joint was closely related to the lead fractures. In conclusion, large joints as the insertion sites of PNS leads should be avoided to prevent lead fractures.

Keywords: Complications; Fracture; Lead; Peripheral nerve; Stimulation.

Neuromodulation techniques such as spinal cord stimulation (SCS) and peripheral nerve stimulation (PNS) are safe and effective treatments for various chronic pain conditions [1-3]. Complications related to SCS and PNS can be classified as hardware-related, programming-related, or biological, with the first being the most common [4]. Hardware-related complications include electrical lead migration, fracture, and malfunction; extension lead disconnection or misconnection; and battery de-

Corresponding author: Ho Sik Moon, M.D., Ph.D.

Department of Anesthesiology and Pain Medicine, Yeouido St. Mary's Hospital, College of Medicine, The Catholic University of Korea, 10 63-ro, Yeoungdeungpo-gu, Seoul 07345, Korea

Tel: 82-2-3779-1268, Fax: 82-2-783-0368

Email: mhsjshgma@gmail.com

ORCID: https://orcid.org/0000-0002-9446-7335

Received: September 20, 2017.

Revised: January 22, 2018

Accepted: March 6, 2018.

Korean J Anesthesiol 2018 October 71(5): 407-410

https://doi.org/10.4097/kja.d.17.00023 pletion, flipping, and recharging problems [4,5]. Among them, lead fracture is rare, especially in PNS $[4,6]$, and is associated with lead location and extension length [7]. We performed PNS on the medial cord and radial nerve near the shoulder joint. The shoulder joint has the widest range of motion in the body, so the electrical lead is vulnerable to damage. We report an electrical lead injury associated with repetitive joint motion, which is an uncommon hardware-related complication in PNS.

\section{Case Report}

In 2015, a 54-year-old man underwent PNS for brachial plexopathy that resulted from a video-assisted thoracoscopic surgery (VATS) for a superior mediastinal schwannoma that was performed eight years earlier. After the VATS, he reported pain in his medial and posterior forearm, especially in areas innervated by the posterior and medial antebrachial cutaneous nerves. He rated his pain intensity as $8-9$ on a 10 -point numeric rating scale (NRS) in which 0 indicates no pain and 10 indicates the worst pain imaginable. Electromyography revealed left brachial plexopathy that mainly involved the medial and posterior cords. The

(c) This is an open-access article distributed under the terms of the Creative Commons Attribution Non-Commercial License (http://creativecommons.org/ licenses/by-nc/4.0/), which permits unrestricted non-commercial use, distribution, and reproduction in any medium, provided the original work is properly cited. 
patient's pain was intractable to various conservative pharmacological and non-pharmacological treatments, so he had received SCS from 2012 to 2015. However, his condition became resistant to this treatment, so it was switched to ultrasound-based PNS that was performed with percutaneous electrical leads (Medtronic, USA). One lead was inserted into the radial nerve via an axillary approach, and another was fixed onto the medial cord via an infraclavicular approach (Fig. 1) [8]. After the

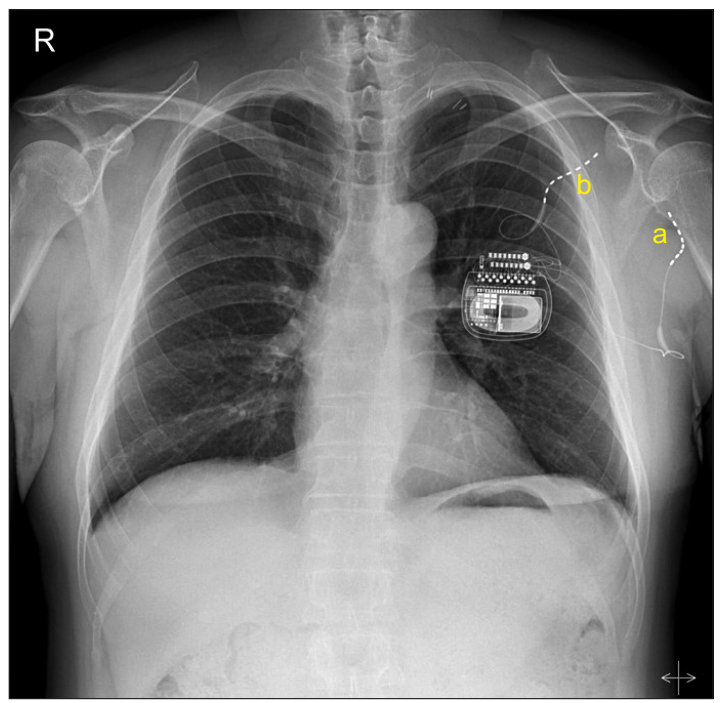

Fig. 1. PNS lead positions prior to lead fractures. One lead was inserted on the radial nerve for pain in the posterior antebrachial cutaneous territory (a), and another was fixed on the medial cord for pain in the medial antebrachial cutaneous dermatome (b). PNS: peripheral nerve stimulation.

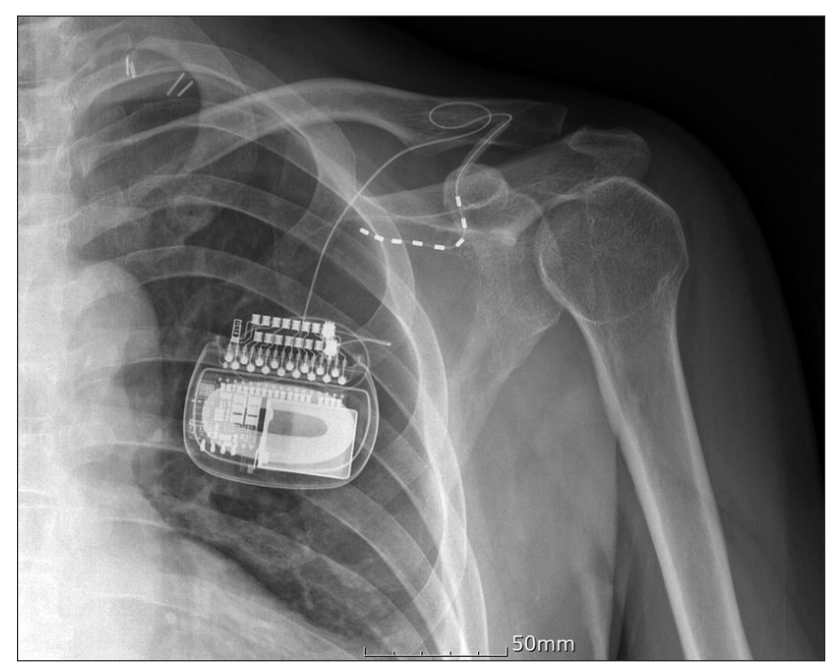

Fig. 2. Lead positions after the PNS revision procedure. A new lead was inserted on the medial cord. The lead was inserted into the trunk side to prevent damage due to joint movement. PNS: peripheral nerve stimulation.
PNS, his NRS-reported pain decreased from 8-9 to 1-2, and he required no analgesics. The PNS provided effective therapy for approximately 20 months, but then the patient's pain returned at an NRS-reported level of 7-8. He reported a frequent electric shock-like sensation at the implantable pulse generator's insertion site, and a physical examination revealed hyperalgesia and mechanical allodynia on his medial and posterior forearm. We confirmed fractures on both electrical leads with N'Vision (Medtronic), a hand-held device for programming Medtronic devices. This device revealed abnormally high electrode impedance $(>10,000 \Omega)$. The fractured leads were removed, and a new lead was inserted onto the medial cord with ultrasound guidance (Figs. 2 and 3). The patient was placed in a supine position with his arm abducted. After obtaining a short-axis view of the axillary artery and vein, we placed a Tuohy needle inplane between the axillary artery and the medial cord and then injected $10 \mathrm{ml}$ of normal saline to provide space for the lead. We turned the Tuohy needle's bevel toward the trunk and gently inserted the new lead until we detected resistance. We then removed the Tuohy needle and adjusted the lead's position with repeated electrical stimulation because the lead was obscured in the ultrasound-images. We inserted only one new lead because it adequately covered all painful areas, and we inserted it at an opposite site of the joint. After this insertion, the patient's NRS-reported pain decreased from 8-9 to 1-2.

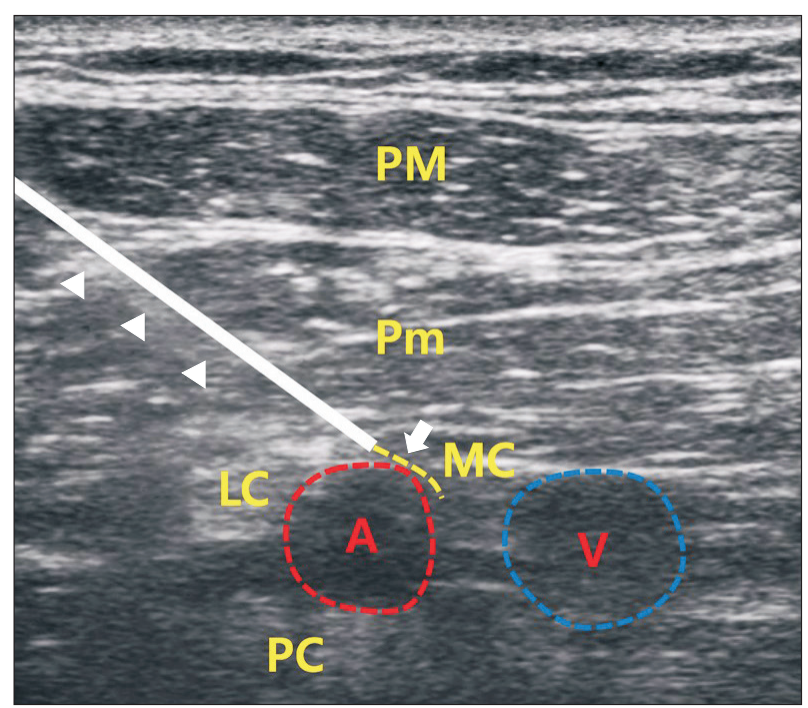

Fig. 3. Ultrasound-guided PNS system implantation. The Tuohy needle (arrow-head) was inserted with a conventional infraclavicular approach, and the needle tip was placed between the axillary artery and the medial cord. After a 10-ml normal saline injection, the lead (arrow) was inserted until resistance was felt. The lead's proper position was settled with repeated electrical stimulation during lead withdrawal. PM: pectoralis major muscle, $\mathrm{Pm}$ : pectoralis minor muscle, MC: medial cord, LC: lateral cord, A: axillary artery, V: axillary vein, PC: posterior cord, PNS: peripheral nerve stimulation. 


\section{Discussion}

PNS is applied to various peripheral nerves, so it involves diverse techniques. Generally, paddle leads or percutaneous leads that are applied for SCS can also be used for PNS. Paddle leads offer many advantages over percutaneous leads. First, undesirable electrical stimulation can be avoided because paddle leads can be precisely and stably arranged over the entire nerve [7]. Additionally, paddle leads have a broader contact area than percutaneous leads do, which enables stimulation of multicomponent nerves such as the sciatic nerve, with its peroneal and tibial components [7]. However, inserting and removing paddle leads necessitates open surgeries under general anesthesia [7], which limits intraoperative physician-patient communication. With regional anesthesia, the physician cannot determine the appropriate stimulation parameters, so this communication with the patient is very important for success in neuromodulation procedures such as SCS and PNS. On the other hand, using percutaneous leads for PNS avoids the need for surgically dissecting nerves under general anesthesia, but percutaneous leads are vulnerable to migration, kinking, and rotation [9]. Moreover, it is difficult to precisely place percutaneous leads over the target nerve, so it is common to make multiple insertion attempts, which can increase the risk of lead damage. In this case, we inserted the percutaneous leads with an ultrasound-guided technique [10], so correctly positioning the leads was not difficult.

A meta-analysis of two randomized controlled studies, two retrospective analyses, and two review articles featuring a combined 4,602 patients calculated a mean lead fracture rate for SCS of $6.37 \%$ (95\% CI: 2.63-10.10\%) [4]. For occipital nerve stimulation and peripheral field stimulation, a meta-analysis of one randomized controlled trial and three retrospective analyses featuring a combined 277 patients calculated a mean lead fracture rate of less than 5\% [4]. However, no such estimates are available for the incidence of lead fracture in PNS applied to the brachial plexus, as in our case.

Generally, the best way to prevent lead damage is to avoid crossing a joint, but this can be challenging for several reasons. First, the target nerves may be inconveniently located, namely PNS may be difficult to perform due to the location of target nerves in certain cases. PNS for upper extremities can be performed at the trunk, division, cord, and terminal branch levels. In this case, we selected the medial cord for stimulating the medial antebrachial cutaneous nerve, which innervates the medial forearm, and the radial nerve for stimulating the posterior antebrachial cutaneous nerve, which innervates the posterior forearm. If we had performed PNS on the proximal brachial plexus at the trunk or division level, we could have avoided the large shoulder joint and covered a larger area. However, this could have provoked unwanted stimuli that would have distressed the patient. Second, post-PNS pain relief increases the joint's motion range. Our patient did not move his shoulder and elbow joints because of severe pain, so we inserted leads near the shoulder joint. However, the implanted leads moved with the shoulder's increased motion range, which may have caused the lead fractures [9].

In conclusion, although PNS is a highly effective and safe treatment, it has a few disadvantages. Among them, lead fracture is a relatively late complication that necessitates repeat operations. To prevent lead fractures resulting from repetitive motion, clinicians should avoid crossing the leads over large joints and implanting leads near such joints if possible.

\section{ORCID}

Shu Chung Choi, https://orcid.org/0000-0001-6565-2112

Ji Seon Chae, https://orcid.org/0000-0002-5914-678X

Youn Jin Kim, https://orcid.org/0000-0001-9189-5839

Jin Young Chon, https://orcid.org/0000-0002-9827-5883

Ho Sik Moon, https://orcid.org/0000-0002-9446-7335

\section{References}

1. Deer TR, Mekhail N, Provenzano D, Pope J, Krames E, Leong M, et al. The appropriate use of neurostimulation of the spinal cord and peripheral nervous system for the treatment of chronic pain and ischemic diseases: the Neuromodulation Appropriateness Consensus Committee. Neuromodulation 2014; 17: 515-50.

2. Park CH, Huh BK. Permanent peripheral nerve stimulation for chronic occipital neuralgia: case reports. Korean J Pain 2008; $21: 155-8$.

3. Chung W, Kim Y, Lee J, Lee S, Kim Y, Shin Y, et al. Ultrasound-guided placement of a permanent peripheral nerve stimulator in a patient with complex regional pain syndrome: A case report. Anesth Pain Med 2016; 11: 295-8.

4. Eldabe S, Buchser E, Duarte RV. Complications of spinal cord stimulation and peripheral nerve stimulation techniques: a review of the literature. Pain Med 2016; 17: 325-36.

5. Sharan A, Huh B, Narouze S, Trentman T, Mogilner A, Vaisman J, et al. Analysis of adverse events in the management of chronic migraine by peripheral nerve stimulation. Neuromodulation 2015; 18: 305-12.

6. Sator-Katzenschlager S, Fiala K, Kress HG, Kofler A, Neuhold J, Kloimstein H, et al. Subcutaneous target stimulation (STS) in chronic noncancer pain: a nationwide retrospective study. Pain Pract 2010; 10: 279-86. 
7. Pope JE, Carlson JD, Rosenberg WS, Slavin KV, Deer TR. Peripheral nerve stimulation for pain in extremities: an update. Prog Neurol Surg 2015; 29: 139-57.

8. Song IA, Gil NS, Choi EY, Sim SE, Min SW, Ro YJ, et al. Axillary approach versus the infraclavicular approach in ultrasound-guided brachial plexus block: comparison of anesthetic time. Korean J Anesthesiol 2011; 61: 12-8.

9. Slavin KV. Technical aspects of peripheral nerve stimulation: hardware and complications. Prog Neurol Surg 2011; 24: 189-202.

10. Kim JH, Shin SH, Lee YR, Lee HS, Chon JY, Sung CH, et al. Ultrasound-guided peripheral nerve stimulation for neuropathic pain after brachial plexus injury: two case reports. J Anesth 2017; 31: 453-7. 